\title{
Macrophages in skin melanoma-the key element in melanomagenesis (Review)
}

\author{
MALGORZATA PIENIAZEK ${ }^{1}$, RAFAL MATKOWSKI ${ }^{2,3}$ and PIOTR DONIZY ${ }^{4}$ \\ ${ }^{1}$ Department of Clinical Oncology, Tadeusz Koszarowski Regional Oncology Centre, Opole 45-061; \\ ${ }^{2}$ Department of Oncology and Division of Surgical Oncology, Wroclaw Medical University, Wroclaw 50-367; \\ ${ }^{3}$ Department of Surgical Oncology, Lower Silesian Oncology Centre, Wroclaw 53-413; ${ }^{4}$ Department of \\ Pathomorphology and Oncological Cytology, Wroclaw Medical University, Wroclaw 50-556, Poland
}

Received August 2, 2017; Accepted November 16, 2017

DOI: $10.3892 / \mathrm{ol} .2018 .8021$

\begin{abstract}
Cutaneous melanoma is an aggressive cancer and its onset and growth are associated, through direct and indirect interactions, with the cancer microenvironment. The microenvironment comprises a dynamic complex of numerous types of cells (due to histogenesis) that constantly interact with each other through multiple cytokines and signaling proteins. Macrophages are one of the most thoroughly studied pleiotropic cells of the immune system. One of their major cytophysiological functions is their involvement in phagocytosis. Previous studies examining the microenvironment of melanomas and tumor-associated macrophages have revealed that they are involved in all stages of melanomagenesis. In the case of cancer initiation, they form an inflammatory microenvironment and then suppress the anticancer activity of the immune system, stimulate angiogenesis, enhance migration and invasion of the cancer cells, and ultimately contribute to the metastatic process. The present review provides a detailed
\end{abstract}

Correspondence to: Dr Malgorzata Pieniazek, Department of Clinical Oncology, Tadeusz Koszarowski Regional Oncology Centre, Katowicka 66a, Opole 45-061, Poland

E-mail: pieniadzgosia@interia.pl

Abbreviations: COX, cyclooxygenase; EGF, epidermal growth factor; EMT, epithelial mesenchymal transition; GM-CSF, granulocyte-macrophage colony-stimulating factor; Il, interleukin; iNOS, inducible nitric oxide synthase; MMP, matrix metalloproteinase; MTF, macrophages with tumor cells fusion; PD-L1, programmed death ligand; PGE2, prostaglandin E2; S1K, sphingosine 1-kinase; STAT3, signal transducer and activator of transcription 3; TAM, tumor-associated macrophage; TGF- $\beta$, transforming growth factor $\beta$; TIC, tumor-initiating cells; TLR, toll-like receptor; TNF, tumor necrosis factor; UPAR, urokinase-type plasminogen activator receptor; VEGF, vascular endothelial cell growth factor

Key words: macrophage, tumor-associated macrophage, melanoma, microenvironment, oncoimmunology overview on the function of macrophages in the melanoma microenvironment.

\section{Contents}

1. Introduction

2. General characteristics of macrophages

3. Macrophages-general function in carcinogenesis

4. Macrophages in skin melanomas

5. Interactions of macrophages with melanoma cells

6. Conclusion

\section{Introduction}

Melanomas are a rare but aggressive cutaneous type of cancer in humans (1). At the dissemination stage in a majority of cases, the disease is resistant to treatment with cytostatics and radiotherapy (1). Therefore, the identification of novel molecular mechanisms involved in the melanomagenesis process and tumor progression have enabled the production of targeted therapies that yield notable effects (1). The basis for melanomagenesis is the accumulation of genetic disorders in the melanocyte (the most frequent ones include the following mutations: B-Raf proto-oncogene, serine/threonine kinase, $\mathrm{N}$-Ras proto-oncogene, GTPase and phosphatase and tensin homolog) (1). However, only the interaction between microenvironment elements and genetic changes in the melanocyte result in the ultimate transformation of a dysplastic melanocyte into a melanoma cell, and at further stages result in the local invasion and dissemination of the primary lesion (1). It is the microenvironment that is one of the key elements of cancer formation and is being studied at present.

A melanoma microenvironment is a markedly heterogenic population of cells that involves fibroblasts, macrophages, lymphocytes, other immune system cells, adipocytes and cells that form the structural elements of cutaneous blood vessels sunk in the extracellular matrix (2). The aforementioned complex network of cellular associations are constantly interacting through direct contact and active protein substances including secretory proteins (e.g., metalloproteinases or 
osteonectin) and growth factors [e.g., transforming growth factor- $\beta$ (TGF- $\beta$ ), Wnt, Hedgehog, epidermal growth factor (EGF), hepatocyte growth factor and platelet-derived growth factor] (2-5), and is accompanied by hypoxia (6).

The present review provides a detailed overview on the function of macrophages in the melanoma microenvironment.

\section{General characteristics of macrophages}

Clonal survival, malignant tumor heterogeneity and resistance to systemic treatment are the features of cancer that, in the light of a previous study, are largely shaped by the immune system (7).

Of all the cells of the immune system, it is the function of macrophages that has been explored most thoroughly. They are a group of cells that are known for their plasticity, which depends on signals from the external environment and thus their cytophysiological functions are widely varied $(8,9)$. There are numerous different propositions on how to divide macrophages. Depending on the immune response, there are three basic groups of macrophages: i) Classically activated macrophages i.e., those being an element of a cellular immune response, produced in the course of inflammatory reaction and formed primarily in response to the granulocyte-macrophage colony-stimulating factor (GM-CSF), interferon- $\gamma$ and tumor necrosis factor (TNF)- $\alpha$, and themselves producing pro-inflammatory cytokines [including interleukin (IL)-12] and reactive forms of oxygen and carbon oxide; they destroy and remove pathogens and abnormal cells and activate other cells of the immune system, ii) wound-healing macrophages which are induced by Il-4 and produce growth factors and proangiogenic factors, and iii) regulatory macrophages which may be produced in response to the excessive release of glucocorticosteroids in stress situations, but which are also induced by the activation of toll-like receptor (TLR) by, among others, the presence of immunoglobulin G complexes; they produce Il-10 which functions as an immunosuppressant, reduces the production of pro-inflammatory factors and inhibits the activity of cytotoxic lymphocytes $\mathrm{T}$ [resulting in the stimulation of programmed death ligand-1 (PD-L1)], and thus limits the inflammatory reaction $(8,9)$. There is also a fourth group of macrophages (trophic macrophages) which phenotypically have the features of macrophages from groups 2 and 3, and are involved in tissue development and the maintenance of homeostasis, regulated primarily by CSF-1 (9).

According to another widely used and much simpler division, macrophages may be grouped as either activated macrophages (M1/activated) or alternatively activated macrophages (M2/trophic) (10). Within each of the groups, macrophage subpopulations are observed, which differ very little with regards to function and phenotype (8). This suggests that macrophages are best understood as a continuum of cells that smoothly transit from one subgroup to another $(8,9)$.

Transcriptional profiling of resident macrophages revealed that the populations are characterized by a high transcriptional variety with minimal overlap, which indicates that there are numerous unique classes of macrophages (11). Heterogeneity of macrophage classes results in a wide range of their biological functions (11). Macrophages are involved in almost all biological processes in an organism, and in addition to their involvement with the immune response to pathogens, they also serve a function in developmental, homeostatic and repair processes (11). Their repair function ensures proper embryogenesis, morphogenesis and organogenesis (11). It was revealed that the loss of macrophages results in a cluster of developmental abnormalities (11). Macrophages are involved in the development of brain, bones, heart and vascular system (11). Additionally, they maintain metabolic homeostasis e.g., in the course of a bacterial infection, they promote resistance to insulin through pro-inflammatory cytokines to decrease nutrient accumulation (11). Macrophages additionally regulate adipocyte responses to insulin (11).

Unfortunately, due to chronic irritation, the notable repair and homeostatic functions of macrophages are lost, which results in their involvement in the development of diseases (11).

\section{Macrophages-general function in carcinogenesis}

Data concerning the function of macrophages in the first stage of initiation and promotion of transformed cancer cells is contradictory. A previous in vitro study confirmed that activated macrophages kill cancer cells (12), whereas other studies reveal that a decreased number of macrophages have no influence on the susceptibility of an organism to cancer; furthermore, in a number of cases, macrophages contribute to the eventual transformation of a given normotypic cell into a cancer cell $(13,14)$. It appears that these differences are due to different macrophage phenotypes and consequently to the production of different cytokines $(9,10)$.

A separate issue is that of a chronic inflammation, in which the activated macrophages serve key functions. It has been hypothesized that the reactive forms of nitrogen and oxygen produce a microenvironment that is conducive to mutagenesis (15). Free radicals damage the DNA of normal cells, and the genome loses stability and results in a transformation into cancer cells (15). Other notable stages in cancer progression include cancer cell invasion, angiogenesis, metastasis and suppression of adaptive anti-tumor immunity $(7,9,16)$. Tumor-associated macrophages (TAMs) are involved in these stages. TAMs are formed from monocytes circulating in the blood (16). With the help of multiple different cytokines including CSF-1, C-C motif chemokine ligand 2 (CCL-2), IL-34, vascular endothelial growth factor A (VEGFA) or $\mathrm{C}-\mathrm{X}-\mathrm{C}$ motif chemokine ligand 12 (CXCL12) produced by cancer cells as they infiltrate the tumor through the vessels (16). They accumulate in the invasive tumor edge, cancer cell/stromal border, central tumor mass, hypoxic/necrotic regions and perivascular areas (16). TAMs have features of a trophic macrophage phenotype (M2), which means that they may remodel the extracellular matrix and suppress the immune system $(8,14,17)$. TAMs produce other mediators and enzymes. Secreted Protein Acidic and Rich in Cystein, cathepsins and metalloproteinase (MMP) 2 and 9 produced by TAMs decompose the elements of the extracellular matrix, including type IV collagen, which is the basic element of the basal membrane, which enables and promotes invasion $(9,11)$. Macrophages additionally regulate angiogenesis and are associated with the density of microvessels surrounding the tumor (16). Proangiogenic macrophages are characterized by the increased expression of tyrosine-protein kinase receptor 2 (TIE2) and the production of VEGF. Their proangiogenic 
function is increased by angiopoietin-2, produced by activated endothelial cells. Subsequently, the EGF-CSF-1 paracrine loop between macrophages and cancer cells with the CXCL12 chemokine promotes the development of the tumor microenvironment of metastasis i.e., the micro-anatomical site regulating the escape of cancer cells from the primary tumor $(8,16)$.

TAMs modulate the immune reaction by enhancing the synthesis of TGF- $\beta$ and prostaglandin E2 (PGE2), in addition to reducing the synthesis of Il-12, Il-18 and the TLR signaling pathway, which results in the reduced activation of other cells of immune response (17). They markedly influence the cytotoxic lymphocytes $\mathrm{T}$ on which they exert a direct effect (by stimulating the synthesis of arginase and nitrogen oxide that inhibit the effect of cytotoxic $\mathrm{T}$ lymphocytes) and an indirect effect (through IL-10 they stimulate monocytes to express the costimulatory molecule PD-L1 which suppresses these lymphocytes, and through the CCL22 chemokine which affects the regulatory $\mathrm{T}$ lymphocytes that additionally inhibit them) $(9,18)$.

Another notable issue is the function of macrophages in metastasis. Primary tumor cells produce chemoattractants (S100A8 and A9) for myeloid cells that settle on the tissues to prepare space for the colonization by cancer cells and create the pre-metastatic niche (9). The settling cancer cells recruit macrophages from the surrounding myeloid cells and these in turn stimulate the growth and further dissemination of the primary tumor cells (9).

\section{Macrophages in skin melanomas}

The presence of macrophages in primary lesions was revealed in cutaneous melanomas (19), uveal melanomas $(20,21)$ and sinonasal melanomas (22). In the case of cutaneous lesions, they are predominantly located in the primary foci, and to a lesser extent in metastatic foci (19). Elevated numbers of macrophages within a melanoma is markedly associated with poor prognosis (23).

Tumorigenesis and growth. Melanoma cells are able to produce multiple factors that modulate the activity of immune response cells. Autocrine factors stimulate melanoma cells to continue non-controlled proliferation, and those with paracrine activity modulate the microenvironment in order for tumor growth and further invasion to be promoted (24). The most notable factors include GM-CSF, CCL2, IL-8/CXCL-8, TGF- $\beta$, IL-1, IL-6 and IL-10, of which GM-CSF and CCL2 have the highest impact on melanoma macrophages (25). GM-CSF inhibits the cytotoxic effect of macrophages (25). However, presently available data from studies on the effect of CCL2 on macrophage recruitment is contradictory. The effect of CCL2 released by melanoma cells is dependent on the extent of its secretion (26). With a high concentration of CCL2, there is a substantial infiltration of the primary lesion by macrophages, primarily by M1, which aims to destroy the tumor (27). However, with the decreased concentration of CCL2, M2 macrophages accumulate and result in the promotion of tumor growth (27). Contrary to the aforementioned studies, it has been proven that the production of CCL 2 by melanoma cells and the associated TAM recruitment results in enhanced angiogenesis (28) and are associated with a more advanced disease (29). It may be that the discrepant results are due to the two-stage influence of CCL2 on tumor growth. It has been revealed that CCL2 and GM-CSF drive angiogenesis firstly by stimulating the expression of hypoxia-inducible factor-1 $\alpha$ and hypoxia response element, and in turn these factors increase the expression of VEGF-A (30). IL-6 produced by melanoma cells additionally promotes the growth of TAMs, which at the subsequent stage induce IL-10 expression that further drives the vicious circle of immunosuppression (25). IL-10 produced by tumor cells induces the expression of the negative costimulatory molecule B7-H4 identified on TAMs, which on contact with T cells inhibits their proliferation and cytokine release (25). B7-H4 was identified in melanoma cells and TAMs (31).

Immune suppression. IL-10 and TGF $\beta$ are produced by TAMs, which inhibit the differentiation of bone marrow cells into dendritic cells, determining their further differentiation into TAMs (25). Furthermore, the combination of factors produced by melanoma-associated macrophages induce the activity of the myeloid-derived suppressor cells that additionally inhibit the response of the immune system (32). It should be noted that within TAMs, similar to melanoma cells, there is a high expression of PD-L1 that helps TAM and regulatory $\mathrm{T}$ lymphocytes to form an immunosuppressive microenvironment $(33,34)$ (Fig. 1A).

The signal transducer and activator of transcription 3 (STAT3) has been identified as the master regulator of a number of the aforementioned factors. STAT3 is a pro-tumorigenic transcription factor. In melanoma, it regulates all facets of the immune response and promotes production of M2 macrophages $(25,35)$. In melanoma, inhibition of the STAT3 pathway results in the increase of inhibition and pro-apoptotic effects against malignant tumor cells. Additionally, the improved recognition of malignant tumor cells by the immune system has been revealed, in addition to improved responses to the anti-tumor cytokine interferon- $\alpha$ (INF- $\alpha$ ) as a result of STAT3 inhibition (35).

Tumor microenvironment modulation. Molecular signals regulating the specific dialogue between melanoma cells and their microenvironment remain unknown. Kinases associated with the membrane lipids of melanoma cells additionally affect the macrophages which form the microenvironment of melanoma. Emerging reports demonstrate that the key function of sphingosine 1-kinase $(\mathrm{S} 1 \mathrm{~K})$ is that it produces a bioactive lipid called lipid sphingosine 1-phosphate (23). Lower expression of S1K results in the reduction of the number of M2 macrophages and increase of the number of M1 macrophages (23), which may be due to the inhibition of cancer progression. This hypothesis, however, requires further study.

Macrophages also modulate the tumor microenvironment through the production of various proteins, enzymes and oxides, thus promoting proliferation, tumor growth and invasion (Fig. 1B) (9,36). Melanoma-associated macrophages have been revealed to have a distorted balance between the production of inducible nitric oxide synthase (iNOS) and arginase (36). Macrophages use arginine to produce NO by iNOS, or to produce ornithine through arginase activity (36). NO is primarily cytotoxic and ornithine production promotes the proliferation of tumor cells (36). It has been demonstrated that 


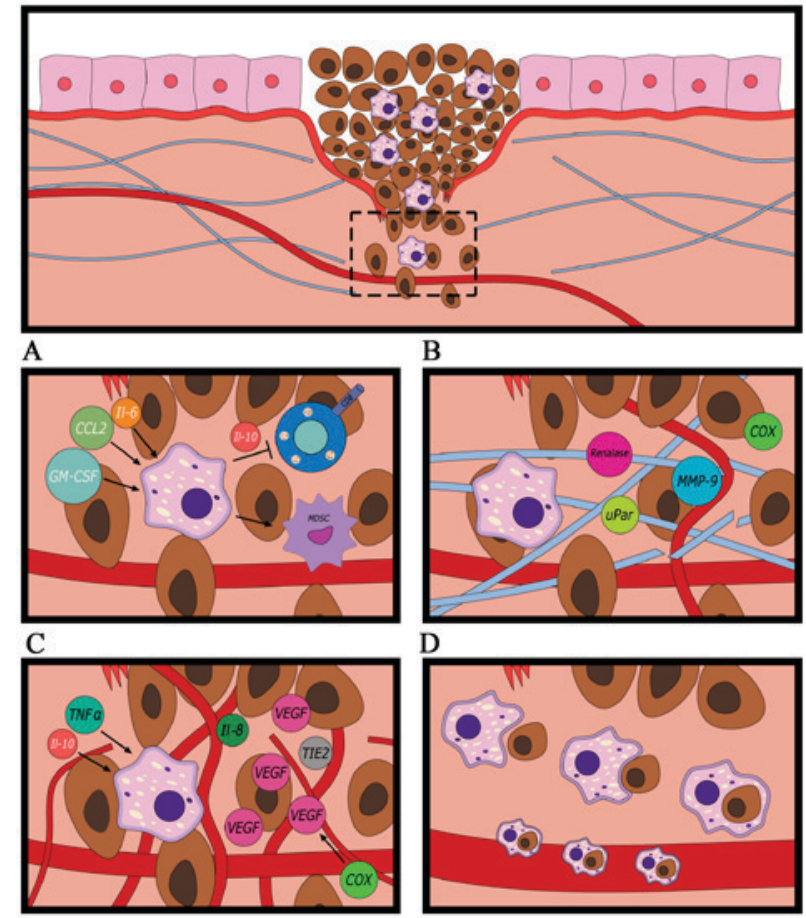

Symbols description:

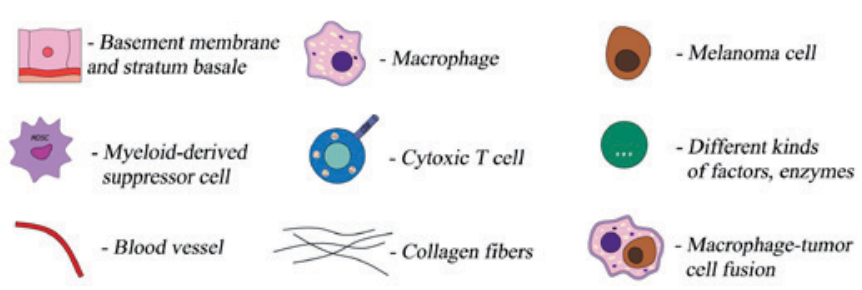

Figure 1. In response to cytokines produced by cancer cells, macrophages transform into TAMs. (A) TAMs modulate the immune reaction, (B) are able to remodel the extracellular matrix (C) promote angiogenesis and (D) contribute to melanoma dissemination. TAM, tumor-associated macrophage; IL, interleukin; CCL, C-C motif chemokine ligand; GM-CSF, granulocyte-macrophage colony-stimulating factor; MMP9 matrix metalloproteinase 9; VEGF, vascular endothelial growth factor; uPar, urokinase-type plasminogen activator receptor; COX, cyclooxygenase; TNF- $\alpha$, tumor necrosis factor- $\alpha$; TIE2, tyrosine-protein kinase receptor 2 .

in clinically less advanced melanomas, iNOS is more active compared with arginase, and this is stimulated by contact with melanoma cells (36). In the case of more advanced primary lesions, a significant reduction in the number of macrophages with a higher percentage of iNOS has been observed (36). It is notable that NO released by macrophages possesses anticancer properties only in the presence of INF $\gamma$ produced by natural killer cells (36).

Melanoma-associated macrophages are characterized by expression of pro-inflammatory protein cyclooxygenase- 2 (COX-2). The percentage of COXs-positive TAMs is highest in in situ and in thin melanomas, and lower in highly advanced and metastatic melanomas. It has been demonstrated that the expression of this thoroughly studied mediator of inflammation is a marker of melanoma progression (19). Macrophages stimulated by osteopontin from the melanoma microenvironment start to synthesize the COX-2 protein (37). $\alpha 9 \beta 1$ integrin on macrophages is the receptor for osteopontin, which stimulates COX-2 expression in macrophages through the ERK and p38 pathways (37). In addition to maintaining inflammation, COX-2 additionally promotes melanoma cell migration and angiogenesis through the COX-2 dependent production of PGE2 (37). Osteopontin combined with PGE2 substantially increases the influence on angiogenesis by enhancing the expression of MMP-9 (36). Another study revealed that the increased expression of COX-2 in melanoma tumor types is associated with increased VEGF expression, density of micro vessels and inflammatory infiltration formed of macrophages $(37,38)$.

Another microenvironment protein that promotes melanoma growth is renalase. This flavoprotein, that functions as cell survival factor, is identified in melanoma cells and cluster of differentiation 163-positive TAMs (39). Renalase activates the phosphoinositide 3-kinase/protein kinase B and mitogen-activated protein kinase signaling pathways which are two of the most important signaling pathways of the epithelial mesenchymal transition (EMT) $(4,39)$. Attenuation of renalase expression reduces melanoma cell survival and inhibits tumor growth (39), which may suggest a potential novel target of molecular targeted anticancer therapies.

TAMs additionally produces protease-like proteins which promote melanoma invasion $(9,24,40)$. It has been demonstrated that melanoma-associated macrophages synthesize large amounts of MMP-9 and urokinase-type plasminogen activator receptor (UPAR) as a result of direct contact with melanoma cells (40). MMP-9 decomposes collagen IV, which is main component of the basement membrane, and additionally decomposes latent TGF- $\beta$ complexes, which further intensifies the EMT $(2,40)$. UPAR is an element of the plasminogen activation system and is involved in multiple proteolytic processes that result in the reorganization and degradation of the extracellular matrix (40).

Angiogenesis. Melanoma-associated macrophages are additionally involved in the indirect promotion of angiogenesis (Fig. 1C) as they release TNF- $\alpha$ and IL- $1 \alpha$. In response to stimulation with these cytokines, melanoma cells produce angiogenic factors, including IL-8, VEGF, TIE2 and CD31, which results in neoangiogenesis $(29,41)$. Furthermore, the proangiogenic factor released by pericytes-milk fat globule-epidermal growth factor 8 stimulates the polarization of M2 macrophages, suggesting that they also increase tumor angiogenesis (42).

Hypoxia is yet another element of the microenvironment notably associated with and affecting TAMs (43). Hypoxia within the tumor drives TAM accumulation in the melanoma microenvironment (43). Signals connecting TAM recruitment with hypoxia have yet to be elucidated. It has been confirmed that high-mobility group box 1 (HMGB1) protein is released by melanoma cells under hypoxic conditions and promotes the accumulation of M2 macrophages and the production of immunosuppressive IL-10 surrounding the tumor (43). HMGB1 stimulates macrophages to produce IL-10, affecting the receptor for advanced glycation end products, thereby inducing an inflammatory response (43).

\section{Interactions of macrophages with melanoma cells}

Interactions of M2 macrophages with the subpopulations of melanoma cells are not well-known. Studies on mice with spontaneous melanoma have revealed that CD34 tumor-initiating cells (TICs) and stem-like cells, i.e., the cells that initiate 
tumor growth and determine certain features of a tumor, including chemoresistance, are M2 macrophage dependent, and the precise survival and proliferation of TICs are dependent on TAMs (44). Furthermore, TICs are stimulated in the presence of TAMs to form melanospheres i.e., non-adherent colonies of melanoma cells (44). It has been demonstrated that CD34- TIC stimulation by TAMs is associated with melanoma progression in vivo (44). The results additionally include a notable observation concerning how chemotherapy was used to treat melanoma (cisplatin, temozolamide), as it was demonstrated that chemotherapy drives TAM recruitment in the tumor, stimulates the growth of TAM-responsive TICs, and that TAMs themselves protect TICs against chemotherapy effects (44). It was demonstrated that on the molecular level, the simulation of TICs was a result of TAM-derived TGF- $\beta$ and polyamines $(44,45)$. The function of TAM-derived TGF- $\beta$ is to autoregulate and stimulate arginase, which results in the production of polyamines (which serve key functions in the growth and differentiation of cancer cells) $(44,45)$.

Dissemination. Another function of melanoma-associated macrophages is their involvement in dissemination (Fig. 1D) which is the main reason for cancer-associated mortality $(5,9,16)$. One of the most widely accepted hypotheses for cancer cell dissemination is the EMT allowing cancer cells to acquire the ability to migrate $(4,5)$. According to an alternative theory, macrophages are involved in the dissemination process. They are said to fuse with tumor cells creating a hybridoma [a macrophage with tumor cell fusion (MTF)] and then pass into the bloodstream (46). MTFs were identified in patients with cutaneous melanoma (46). The selected MTFs presented with features of macrophages and melanomas. Morphologically, MTFs were large cells with pseudopodia and lamellipodia. Expression of macrophage characteristic markers (CD14 and CD68) in addition to M2 macrophage-specific markers (CD163, CD204, CD206) were identified (46). Expression of characteristics of melanocytes [including activated leukocyte cell adhesion molecule, protein melan-A and pro-carcinogenic cytokine macrophage migration inhibitory factor (MIF)] were additionally demonstrated (46). In one case MTFs possessed a B-Raf proto-oncogene, serine/threonine kinase mutation (44). These results suggest that macrophages are involved in melanoma dissemination through the formation of MTFs which may escape to the bloodstream and are able to settle in distant organs, and the released cytokines (including MIF) prepare niches to be colonized by the TICs (46). Further studies demonstrated that the endothelial cells next to the melanoma produce an increased number of various chemokines including CCL21, CCL2 and CXCL8 $(16,47)$. They result in the endothelium becoming permeable, melanoma cells expressing receptors for these chemokines intravasating, and then through the blood reaching the pre-metastatic niches (16). It may be assumed that similarly to breast cancer, they form micro-clots with blood platelets there and remain in the blood vessels (16). Pre-metastatic niches are a reservoir of monocytes recruited from the circulatory system which then transform to metastatic associated macrophages (16). They promote metastatic cell survival by adhesion and survival signal (namely, CCL3) (16).

\section{Conclusion}

As presented in this review, macrophages serve a key function in the complex regulation of the network of interactions and associations between melanoma cells and multiple subpopulations of cells that form the tumor stroma. Improved knowledge of the macrophages, namely the identification of their phenotypes and their influence on promoting all stages of melanomagenesis, has altered our perception of the macrophages. They are no longer involved exclusively in phagocytosis and the specific clearance of dead cells, but they have become a notable and active element of melanomagenesis. Furthermore, the knowledge of signaling pathways, protein substances and their ligands paved the way for breakthrough targeted therapies against disseminated melanoma (e.g., using an anti-PD-L1 antibody) (12). Targeted therapies may be directly aimed at TAMs to eliminate them or modulate their activity, and alternatively they may be used to support the existing treatment methods. It has been revealed that activation of macrophages induces their activity against melanoma (48). It may be achieved by administering immunomodulatory substances including GM-CSF, galectin-9, vaccination with pathogens, nanoparticles (polyhydroxylated fullerenols) or blocking melanoma inhibition of macrophage migration by a macrophage inhibitory cytokine inhibitor (48). Another therapeutic strategy is to prevent the transformation of macrophages into TAMs. For this strategy, antibodies neutralizing Il-4, Il-10 or TGF- $\beta$ may be used (48). Finally, TAM-targeted therapies are being studied. There are encouraging preclinical studies of inhibitor STAT-3, janus kinase-2 (35) or nanoparticles delivering small interfering RNA to TAMs (49). Multifunctional TAMs appear to be an attractive anti-melanoma target, which may complete other treatments as part of an effective and comprehensive anti-melanoma strategy.

\section{Acknowledgements}

The presented results of studies performed as part of the subject as per records in the Simple system number ST.C280.17.010 were financed through a statutory subsidy by the Minister of Science and Higher Education.

\section{References}

1. Lin K, Baritaki S, Militello L, Malaponte G, Bevelacqua Y and Bonavida B: The role of B-RAF mutations in melanoma and the induction of EMT via dysregulation of the NF- $\kappa$ B/Snail/RKIP/PTEN circuit. Genes Cancer 1: 409-420, 2010.

2. Taylor MA, Parvani JG and Schiemann WP: The pathophysiology of epithelial-mesenchymal transition induced by transforming grow th factor-beta in normal and malignant mammary epithelial cells. J Mammary Gland Biol Neoplasia 15: 169-190, 2010.

3. RuiterD, Bogenrieder T, Elder D and Herlyn M: Melanoma-stroma interactions: Structural and functional aspects. Lancet Oncol 3: 35-43, 2002.

4. Kalluri R and Weinberg RA: The basics of epithelial-mesenchymal transition. J Clin Invest 119: 1420-1428, 2009.

5. Wu Y and Zhou BP: New insights of epithelial-mesenchymal transition in cancer metastasis. Acta Biochim Biophys Sin (Shanghai) 40: 643-650, 2008.

6. Bedogni B and Powell MB: Hypoxia, melanocytes and melanoma-survival and tumor development in the permissive microenvironment of the skin. Pigment Cell Melanoma Res 22: 166-174, 2009. 
7. Palucka AK and Coussens LM: The basis of oncoimmunology Cell 164: 1233-1247, 2016.

8. Mosser DM and Edwards JP: Exploring the full spectrum of macrophage activation. Nat Rev Immunol 8: 958-969, 2008.

9. Qian BZ and Pollard JW: Macrophage diversity enhances tumor progression and metastasis. Cell 141: 39-51, 2010.

10. Mantovani A and Sica A: Macrophages, innate immunity and cancer: Balance, tolerance, and diversity. Curr Opin Immunol 22: 231-237, 2010

11. Wynn TA, Chawla A and Pollar JW: Macrophage biology in development, homeostasis and disease. Nature 496: 445-455, 2013.

12. Klimp AH, de Vries EG, Scherphof GL and Daemen T: A potential role of macrophage activation in the treatment of cancer. Crit Rev Oncol Hematol 44: 143-161, 2002.

13. Teng MW, Swann JB, Koebel CM, Schreiber RD and Smyth MJ Immune-mediated dormancy: An equilibrium with cancer. J Leukoc Biol 84: 988-993, 2008.

14. Lin EY and Pollard JW: Tumor-associated macrophages press the angiogenic switch in breast cancer. Cancer Res 67: 5064-5066, 2007.

15. Pang B, Zhou X, Yu H, Dong M, Taghizadeh K, Wishnok JS, Tannenbaum SR and Dedon PC: Lipid peroxidation dominates the chemistry of DNA adduct formation in a mouse model of inflammation. Carcinogenesis 28: 1807-1813, 2007.

16. Lewis CE, Harney AS and Pollard JW: The multifaceted role of perivascular macrophages in tumors. Cancer Cell 30: 365, 2016.

17. Torroella-Kouri M, Silvera R, Rodriguez D, Caso R, Shatry A, Opiela S, Ilkovitch D, Schwendener RA, Iragavarapu-Charyulu V, Cardentey $\mathrm{Y}$, et al: Identification of a subpopulation of macrophages in mammary tumor-bearing mice that are neither M1 nor M2 and are less differentiated. Cancer Res 69: 4800-4809, 2009.

18. Curiel TJ, Coukos G, Zou L, Alvarez X, Cheng P, Mottram P, Evdemon-Hogan M, Conejo-Garcia JR, Zhang L, Burow M, et al Specific recruitment of regulatory $\mathrm{T}$ cells in ovarian carcinoma fosters immune privilege and predicts reduced survival. Nat Med 10: 942-949, 2004

19. Bianchini F, Massi D, Marconi C, Franchi A, Baroni G, Santucci M, Mannini A, Mugnai G and Calorini L: Expression of cyclo-oxygenase-2 in macrophages associated with cutaneous melanoma at different stages of progression. Prostaglandins Other Lipid Mediat 83: 320-328, 2007.

20. Bronkhorst IH, Ly LV, Jordanova ES, Vrolijk J, Versluis M, Luyten GP and Jager MJ: Detection of M2 macrophages in uveal melanoma and relation with survival. Invest Ophthalmol Vis Sci 52: 643-650, 2011

21. Jager MJ, Ly LV, El Filali M and Madigan MC: Macrophages in uveal melanoma and in experimental ocular tumor models: Friends or foes? Prog Retin Eye Res 30: 129-146, 2011.

22. Shi L, Lei D, Ma C, Xu F, Li Y, Wang Y, Cong N, Liu D and Pan XL: Clinicopathological implications of tumour-associated macrophages and vascularization in sinonasal melanoma. J Int Med Res 38: 1276-1286, 2010

23. Mrad M, Imbert C, Garcia V, Rambow F, Therville $N$, Carpentier S, Ségui B, Levade T, Azar R, Marine JC, et al: Downregulation of sphingosine kinase-1 induces protective tumor immunity by promoting M1 macrophage response in melanoma. Oncotarget 7: 71873-71886, 2016.

24. Lázár-Molnár E, Hegyesi H, Tóth S and Falus A: Autocrine and paracrine regulation by cytokines and growth factors in melanoma. Cytokine 12: 547-554, 2000.

25. Ilkovitch D and Lopez DM: Immune modulation by melanoma-derived factors. Exp Dermatol 7: 977-985, 2008.

26. Graves DT, Barnhill R, Galanopoulos T and Antoniades HN Expression of monocyte chemotactic protein-1 in human melanoma in vivo. Am J Pathol 140: 9-14, 1992.

27. Nesbit M, Schaider H, Miller TH and Herlyn MJ: Low-level monocyte chemoattractant protein-1 stimulation of monocytes leads to tumor formation in nontumorigenic melanoma cells. J Immunol 166: 6483-6490, 2001.

28. Gazzaniga S, Bravo AI, Guglielmotti A, van Rooijen N, Maschi F, Vecchi A, Mantovani A, Mordoh J and Wainstok R: Targeting tumor-associated macrophages and inhibition of MCP-1 reduce angiogenesis and tumor growth in a human melanoma xenograft. J Invest Dermatol 127: 2031-2041, 2007.

29. Torisu H, Ono M, Kiryu H, Furue M, Ohmoto Y, Nakayama J, Nishioka Y, Sone S and Kuwano M: Macrophage infiltration correlates with tumor stage and angiogenesis in human malignant melanoma: Possible involvement of TNFalpha and IL-1alpha. Int J Cancer 85: 182-188, 2000.
30. Varney ML, Olsen KJ, Mosley RL and Singh RK: Paracrine regulation of vascular endothelial growth factor-a expression during macrophage-melanoma cell interaction: Role of monocyte chemotactic protein-1 and macrophage colony-stimulating factor. J Interferon Cytokine Res 25: 674-683, 2005.

31. Swatler J and Kozłowska E: Immune checkpointtargeted cancer immunotherapies. Postepy Hig Med Dosw (Online) 70: 25-42, 2016 (In Polish).

32. Mao Y, Poschke I, Wennerberg E, Pico de Coaña Y, Egyhazi Brage S, Schultz I, Hansson J, Masucci G, Lundqvist A and Kiessling R: Melanoma-educated CD14+ cells acquire a myeloid-derived suppressor cell phenotype through COX-2-dependent mechanisms. Cancer Res 73: 3877-3887, 2013.

33. Hino R, Kabashima K, Kato Y, Yagi H, Nakamura M, Honjo T, Okazaki T and Tokura Y: Tumor cell expression of programmed cell death-1 ligand 1 is a prognostic factor for malignant melanoma. Cancer 116: 1757-1766, 2010.

34. Kakizaki A, Fujimura T, Furudate S, Kambayashi Y, Yamauchi T, Yagita $\mathrm{H}$ and Aiba S: Immunomodulatory effect of peritumorally administered interferon-beta on melanoma through tumor-associated macrophages. Oncoimmunology 4: e1047584, 2015.

35. Lesinski GB: The potential for targeting the STAT3 pathway as a novel therapy for melanoma. Future Oncol 9: 925-927, 2013.

36. Massi D, Marconi C, Franchi A, Bianchini F, Paglierani M, Ketabchi S, Miracco C, Santucci M and Calorini L: Arginine metabolism in tumor-associated macrophages in cutaneous malignant melanoma: Evidence from human and experimental tumors. Hum Pathol 38: 1516-1525, 2007.

37. Kale S, Raja R, Thorat D, Soundararajan G, Patil TV and Kundu GC: Osteopontin signaling upregulates cyclooxygenase-2 expression in tumor-associated macrophages leading to enhanced angiogenesis and melanoma growth via $\alpha 9 \beta 1$ integrin. Oncogene 33: 2295-2306, 2013.

38. Gregório H, Raposo TP, Queiroga FL,Prada J and Pires I: Investigating associations of cyclooxygenase-2 expression with angiogenesis, proliferation, macrophage and T-lymphocyte infiltration in canine melanocytic tumours. Melanoma Res 26: 338-347, 2016.

39. Hollander L, Guo X, Velazquez H, Chang J, Safirstein R, Kluger H, Cha $\mathrm{C}$ and Desir GV: Renalase expression by melanoma and tumor-associated macrophages promotes tumor growth through a STAT3-mediated mechanism. Cancer Res 76: 3884-3894, 2016.

40. Marconi C, Bianchini F, Mannini A, Mugnai G, Ruggieri S and Calorini L: Tumoral and macrophage uPAR and MMP-9 contribute to the invasiveness of B16 murine melanoma cells. Clin Exp Metastasis 25: 225-231, 2008.

41. Kim OH, Kang GH, Noh H, Cha JY, Lee HJ, Yoon JH, Mamura M, Nam JS, Lee DH, Kim YA, et al: Proangiogenic TIE2(+)/CD31 (+) macrophages are the predominant population of tumor-associated macrophages infiltrating metastatic lymph nodes. Mol Cells 36: 432-438, 2013.

42. Yamada K, Uchiyama A, Uehara A, Perera B, Ogino S, Yokoyama Y, Takeuchi Y, Udey MC, Ishikawa O and Motegi S: MFG-E8 drives melanoma growth by stimulating mesenchymal stromal cell-induced angiogenesis and M2 polarization of tumor-associated macrophages. Cancer Res 76: 4283-4292, 2016.

43. Huber R, Meier B, Otsuka A, Fenini G, Satoh T, Gehrke S, Widmer D, Levesque MP, Mangana J, Kerl K, et al: Tumour hypoxia promotes melanoma growth and metastasis via high mobility group box-1 and M2-like macrophages. Sci Rep 6: 29914, 2016.

44. Tham M, Tan KW, Keeble J, Wang X, Hubert S, Barron L, Tan NS, Kato M, Prevost-Blondel A, Angeli V and Abastado JP: Melanoma-initiating cells exploit M2 macrophage TGF $\beta$ and arginase pathway for survival and proliferation. Oncotarget 5: 12027-12042, 2014.

45. Chang CI, Liao JC and Kuo L: Macrophage arginase promotes tumor cell growth and suppresses nitric oxide-mediated tumor cytotoxicity. Cancer Res 61: 1100-1106, 2001.

46. Clawson GA, Matters GL, Xin P, Imamura-Kawasawa Y, Du Z, Thiboutot DM, Helm KF, Neves RI and Abraham T: Macrophage-tumor cell fusions from peripheral blood of melanoma patients. PLoS One 10: e0134320, 2015.

47. Somasundaram R and Herlyn D: Chemokines and the microenvironment in neuroectodermal tumor-host interaction. Semin Cancer Biol 19: 92-96, 2009.

48. Wang H, Zhang L, Yang L, Liu C, Zhang Q and Zhang L: Targeting macrophage anti-tumor activity to suppress melanoma progression. Oncotarget 8: 18486-18496, 2017.

49. Qian Y, Qiao S, Dai Y, Xu G, Dai B, Lu L, Yu X, Luo Q and Zhang Z: Molecular-targeted immunotherapeutic strategy for melanoma via dual-targeting nanoparticles delivering small interfering RNA to tumor-associated macrophages. ACS Nano 11: 9536-9549, 2017. 